\title{
Secure and Scalable Data Collection with Time Minimization in the Smart Grid
}

\author{
Suleyman Uludag ${ }^{1}$, King-Shan Lui $^{2}$, Wenyu Ren $^{3}$, and Klara Nahrstedt ${ }^{3}$ \\ ${ }^{1}$ Department of Computer Science, University of Michigan - Flint, MI, USA \\ ${ }^{2}$ Department of Electrical and Electronic Engineering, The University of Hong Kong, Hong Kong \\ ${ }^{3}$ Department of Computer Science, University of Illinois at Urbana-Champaign, IL, USA
}

\begin{abstract}
Deployment of data generation devices, such as sensors and smart meters, has been accelerating towards the vision of Smart Grid. The volume of data to be collected increases tremendously. Secure, efficient, and scalable data collection becomes a challenging task. In this paper, we present a secure and scalable data communications protocol for Smart Grid data collection. Under a hierarchical architecture, relay nodes (aka data collectors) collect and convey the data securely from measurement devices to the power operator. While the data collectors can verify the integrity, they are not given access to the content, which may pave the way for third party providers to deliver value-added services or even the data collection itself. We further present optimization solutions for minimizing the total data collection time.
\end{abstract}

\section{INTRODUCTION}

In the Smart Grid, massive number of sensors or measurement devices will be installed to collect real-time information. The generated data should be collected in a secure, efficient, and scalable manner. To make it scalable, a hierarchical data collection framework is usually adopted. For example, in Advanced Metering Infrastructure (AMI), smart meters first report measurements to data concentrators [1]. Thereby, the power operator does not have to maintain a separate, expensive connection with each smart meter. Apart from data collection, this hierarchical communication structure should also allow the power operator to send an instruction to the devices. To maintain fast response, messages, data or instructions, should be delivered efficiently and as fast as possible. The messages should be protected to prevent from information leak and launch of attacks. In this paper, we develop a comprehensive protocol that allows a power operator to collect data, as well as send commands to measurement devices in a secure, scalable, and efficient manner.

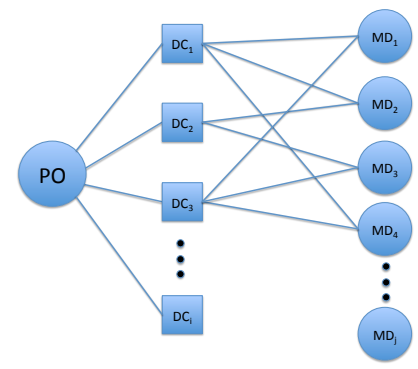

Fig. 1. Hierarchical Data Collection Structure.

Fig. 1 presents the data collection architecture considered in this paper. The Measurement Devices (MDs) are sensors or smart meters that generate power-grid specific data. They are small telemetric devices and computationally constrained. Each MD is connected to at least one Data Collector (DC), and each DC may connect to multiple MDs. The Power Operator (PO) has a direct connection with each DC. PO and DCs are relatively more powerful than MDs. The data are reported to PO via a set of DCs. PO may also issue commands to the MDs via the DCs. Theoretically, a DC is trustworthy if it is within the security domain of the PO.

However, due to the massive number of MDs and their dispersion over a large area, it may not be appropriate to assume DCs can be completely trusted. In addition, one of the seven actors identified by The National Institute of Standards and Technology (NIST) in the Smart Grid (SG) Framework [2] is third party service providers which are to furnish valueadded services. We assume honest-but-curious model for DCs. Thus, the data collection tasks may be outsourced to third party service providers [3]. Besides, the benefits of cloud computing [4] may be accrued for storage and processing of the data collected. Data sharing to others to provide services like energy management services can be facilitated as well.

In some other applications [5], DCs are mobile and the connections between DCs and MDs are dynamic. Therefore, it would be desirable for MDs to encrypt their data in a way that DCs do not have access to them. In other words, each MD should encrypt its data using an appropriate key to keep its data private from DCs and other possible adversaries. On the other hand, due to limitation in memory and computational capability, the encryption algorithm used should be efficient. PO should also protect its commands appropriately. Apart from ensuring the security of these commands, it is also crucial to deliver data and commands promptly because fast actions of MDs are necessary to maintain the stability and health of the smart grid. Because the network delay for different DCs to collect data from a certain MD would be different, to make the data collection more efficient, we also study how to assign DCs to MDs to minimize the time for data collection.

Our contributions in this paper may be summarized as follows:

- Under a hierarchical infrastructure, we have proposed a scalable, secure, and lightweight data collection scheme in the Smart Grid, 
- Our secure data collection scheme does not assume trusted DCs, rather they are considered to be honest-butcurious entities. This may pave the way for outsourcing the data collection and third party service providers as envisioned by the NIST's Smart Grid Framework [2],

- We have coupled the secure date collection with an optimization problem formulation with the objective function of time minimization, which is a first in the literature as a joint security and optimization approach,

- We have shown the NP-Hardness of the problem and developed an efficient heuristic solution,

- We have also provided a solution to the assignment of DCs to MDs as part of the optimization framework.

The rest of the paper is organized as follows: Sec. II describes existing efforts on data collection in smart grids. We provide the system and protocol overview in Sec. III. The details of the protocol are described in Sec. IV and V. In Sec. VI, we analyze the time performance of our mechanism and present the DC-MD assignment problem as an optimization problem. We conclude our paper in Section VIII.

\section{RELATED WORK}

Data integrity and confidentiality of end-to-end data have been studied extensively in the Internet. However, most schemes, such as TLS [6], assume the devices have abundant memory and computational power to perform expensive cryptographic operations. In smart grids, on the other hand, reporting devices have limited memory with a slow CPU. Traditional Internet security protocols are thus not suitable for data collection in smart grids [7].

DNP3 (Distributed Network Protocol) [8] is a standard communications protocol used in SCADA (Supervisory Control And Data Acquisition), the data collection subsystem of power grids. It assumes all components are within the security perimeter of the operator and is not designed to protect data forwarded by the DC as in our situation. A more recent standard for substation automation is the IP-based IEC (the International Electrotechnical Commission) 61850 [9]. Yet, IEC 61850 was also initially designed without security mechanisms [10]. It is thus generally agreed by the experts that new security protocols for data collection and command delivery of smart grids need to be developed.

Our proposed approach comprises security aspect of the smart grid data collection as well as the time minimization. In what follows, we provide synopses of related work from these aspects as well as with respect to a variety of other relevant subtopics of our holistic approach.

Collecting data by means of transport layer protocols from a massive number of MDs has been studied in the literature as an option. [11] studies how to reduce the storage needed when the control center needs to establish multiple sessions with the MDs. Long-term shared keys are generated by a function so that the control center only needs to memorize the function but not individual keys. Nevertheless, the key developed this way is not very secure. Besides, the protocol is not suitable for the hierarchical data collection architecture.
Data collection through a data collector is considered in [12]. The authors propose to maintain two separate Transmission Control Protocol (TCP) connections, and the two connections can be protected using different mechanisms independently. Nevertheless, the data collector is assumed to be trustworthy, that is, it can read the data sent by the MD.

Another important aspect of secure data collection is concerned with key management. Many assume trusted DC, that is, they do not consider hiding the data from the DCs, such as [13], [14], [15]. Some others assume a direct connection between the PO and MDs [16]-[18] and some others are not suitable for hierarchical data collection as we consider in this paper [16], [17], [19].

The SAKE protocol [20] allows two neighboring sensor nodes to establish keys using hash chains. However, the authors assume the attackers are of limited computational capability as another sensor. The authors in [15] apply the elliptic curve public key technique to perform key management. Mutual authentication between different entities is studied. Nevertheless, there is no discussion on how to protect the data reported by a sensor.

Some protocols have been developed to establish shared keys when the two parties can establish direct communication. [18] describes how to establish keys and secure unicast and multicast communications. [7] proposes long-term keys to be given to the different parties for protecting messages. [16] describes how to apply the Diffie-Hellman mechanism to establish a shared key for data authentication between two parties. [17], on the other hand, relies on identity-based cryptography. All these mechanisms cannot be applied in the hierarchical data collection model because the PO and the MDs cannot establish a direct connection. The authors in [21] describe how a device establishes shared keys with different controllers at different hierarchical levels. However, it is assumed that a shared key exists between two adjacent controllers. Another approach presented in [22] is based on symmetric cryptography to provide data confidentiality and authentication between sensors and the base station. Again, a master key is assumed with a pre-agreed pseudo-random function in the scheme.

Another category for providing security and privacy [23] exploits the aggregate statistics of the sensed data, such as summation, average, minimum, maximum, etc. These approaches take advantage of in-network data processing (also referred to as aggregation) to induce some obfuscating operations on the transmitted data [24]-[31]. Examples of this category include cluster-based private data aggregation [24] and its integrity enhanced version [25], secret perturbation [26], kindistinguishable privacy-preserving data aggregation [27], a centralized authentication server based in-network aggregation for AMI [28], [29], a secure architecture for distributed aggregation of additive data [30], and a network codingbased encryption between smart meters and aggregators [31]. Unlike these techniques, our problem formulation does not assume any statistical property for in-network processing and deliver the MD data unaltered to the PO. There are also 
data aggregation schemes without any security schemes, such as [32], [33].

There are also homomorphic encryption-based approaches to hide the collected information from the MDs, such as [34]-[39]. However, homomorphic encryption is a computeintensive operation.

[40] studies how data generators report data to a honestbut-curious storage center for a user to retrieve later. To the best of our knowledge, the data collection trust model assumed in this paper is the most related to our scenario. The storage center is similar to the DC in our model that it is semi-trusted, and data should be hidden from it. MDs in our model are the data generators, while PO is a user in their model. However, the paper suggests to use expensive attributebased and public key encryption to protect data to incorporate policy consideration. The experimental computational time for a decryption on a message of size less than 1000 bits in a lowend smart meter (TinyPBC library on a 32-bit ARM XScale PXA271 processor) is around $140 \mathrm{~ms}$, while the encryption is supposed to be a few times more expensive. Our protocol, on the other hand, encrypts data using the much more lightweighted symmetric key cryptography, which is more suitable for computationally-constrained MDs.

There are some approaches with optimization for the data collection process. Cost minimization of data collection by means of wireless channel selection and transmission scheduling has been reported in [41]. A delay minimization of overhead transmission lines over unreliable wireless links is studied in [42]. These and other similar approaches lack any security mechanism as part of their approaches, unlike our proposal in this paper.

An interesting approach in [43] considers the tradeoff between the strength of security and energy consumption jointly for both Phasor Maeasurement Unit (PMU) and AMI data over energy-constrained devices. However, only a generic comparison of different cryptographic algorithms over CrossBow and Ember sensor platforms is reported without any attention to the overall data collection scheme.

To the best of our knowledge, no other paper in the literature appears to be proposing a holistic approach for hierarchical data collection with curious-but-honest DCs with a joint goal of minimizing the overall data collection time and assignment of MDs to DCs. Further, we also perform experiments to study the time performance of our mechanism.

\section{System And Protocol OVERVIEW}

\section{A. Operations and their requirements}

As mentioned in Section I, our communication architecture supports MDs to report data and PO to deliver commands in a timely and secure manner. Table I describes each operation. Op 1 is a regular call-for-data from the PO which is performed periodically. Op 2 is performed when PO detects something abnormal and would like a data report from a particular MD. Time is more critical than a regular data reporting. Op 3 is done when MD detects something abnormal and would like

\begin{tabular}{|c|c|c|c|}
\hline & Operation & $\begin{array}{l}\text { Security } \\
\text { Requirement }\end{array}$ & Time Requirement \\
\hline Op 1 & $\begin{array}{l}\text { PO initiates } \\
\text { data collection } \\
\text { of all MDs } \\
\text { or a group of } \\
\text { MDs }\end{array}$ & $\begin{array}{l}\text { Data reported should } \\
\text { be authenticated and } \\
\text { should be read only } \\
\text { by the PO, not by } \\
\text { other MDs or any DC }\end{array}$ & $\begin{array}{l}\text { The total time to col- } \\
\text { lect all data should } \\
\text { be minimized }\end{array}$ \\
\hline Op 2 & $\begin{array}{l}\text { PO requests } \\
\text { data from a } \\
\text { certain } \mathrm{MD}\end{array}$ & Same as Op 1 & $\begin{array}{l}\text { The time needed } \\
\text { should be kept } \\
\text { minimal }\end{array}$ \\
\hline Op 3 & $\begin{array}{l}\text { MD initiates } \\
\text { an urgent data } \\
\text { report }\end{array}$ & Same as Op 1 & $\begin{array}{l}\text { The data should be } \\
\text { delivered to the PO } \\
\text { as soon as possible }\end{array}$ \\
\hline Op 4 & $\begin{array}{l}\text { PO issues } \\
\text { an urgent } \\
\text { command to a } \\
\text { group of MDs }\end{array}$ & $\begin{array}{l}\text { The command should } \\
\text { be authenticated ap- } \\
\text { propriately }\end{array}$ & $\begin{array}{l}\text { Time for each MD to } \\
\text { receive and read the } \\
\text { command should be } \\
\text { minimized }\end{array}$ \\
\hline
\end{tabular}

TABLE I

SYSTEM OPERATIONS AND THEIR REQUIREMENTS.

to report to the PO. OP 4 is issued when PO needs a group of MDs to perform a certain action as soon as possible.

We develop our protocol to be secure from outsider attacks such as eavesdropping, impersonation, and message tampering, etc. There are three types of insiders in the protocol: PO, DCs, and MDs. They are all given with a corresponding pair of public and private key pair (see Section III-B). Similar to other secure communication protocols, we basically assume a signature, which is created by the private key, can be an identity authenticator. That is, if somebody can prove that she has the knowledge of Alice's private key, we assume this person is Alice. If the working environment is very insecure that even private keys could be stolen easily, our protocol does not work. The system, in this case, probably require another form of authentication such as token-based, bio-metric, instead of key-based.

We assume the PO (the entity that possesses the private key of the PO) is always trustworthy because it is the control of the whole system and it decides how to use the data collected. The DCs, on the other hand, are honest-but-curious that they would follow the protocol as specified but would like to read the data and share with others if they could. That is, they would not impersonate another entity in the system, nor actively tamper the data, but would like to learn as much as possible based on the information they can access according to the normal operation of the protocol. As the MDs are devices located in the field (for example, on power grid poles), it is not likely they are under the same physically secure environment as the PO. The chance that leaking private keys is higher. When an attacker gets the private key of a certain MD, it can report fake data to the PO on behalf of the MD. The PO can analyze the data reported to detect whether they were legitimate data or not. Even though this attacker can report fake data on behalf of its victim, it cannot impersonate other entities (other MDs, PO, or DCs) and read the data sent by other MDs.

\section{B. System Parameters}

Before any communication, PO, DCs, and MDs are equipped with a set of system parameters. We assume necessary parameters are configured in a DC or MD before they 
are installed in the field.

1) Long-term keys: We assume there is a key server that can generate a set of public and private keys for each entity in the system. The public/private key pair is configured into a DC or MD before it is installed in the field. PO, on the other hand, apart from keeping its own key pair, it also remembers the public keys of all MDs and DCs in the system. We denote the public key and private key of node $\mathrm{A}$ as $A^{+}$and $A^{-}$, respectively. Under normal circumstances, $\mathrm{PO}$ would not publish the public keys of DCs and MDs to the general public. However, our protocol is secure even if the attackers know the public key information of any DC or MD they want to attack.

2) Diffie-Hellman $(\mathrm{DH})$ parameters: We adopt the DiffieHellman key exchange mechanism to develop shared keys between two parties. Due to space limitation, we refer readers to [44] for the details. Generally speaking, DH allows the two parties to develop a secret shared key even eavesdroppers can read the half keys they exchange with each other. Through forgetting half keys and shared keys appropriately, DH keys also support perfect forward secrecy.

\section{Cryptographic functions}

To provide authentication, confidentiality, integrity, and other security protections, messages have to be encrypted, hashed, or signed. We assume the PO selects appropriate cryptographic algorithms for the purposes, and these functions are installed in the DCs and MDs. For example, PO may use the Advanced Encryption Standard (AES) for symmetric key encryption and SHA-256 (the Secure Hash Algorithm with 256 bits of key length) for hash computation. Table II summarizes the functions used in the protocol. In the table, PKE is Public Key Encryption, PKD is Public Key Decryption, SKE is Symmetric Key Encryption, SKD is Symmetric Key Decryption, $\mathrm{SIGV}$ is signature verification, $K_{p}$ is a public key while $K_{s}$ is a shared key.

\begin{tabular}{|l|l||l|l|}
\hline Name & Description & Name & Description \\
\hline \hline$P K E\left(K_{p}, M\right)$ & encrypt $M$ using $K_{p}$ & $P K D\left(K_{p}, C\right)$ & decrypt $C$ using $K_{p}$ \\
\hline$S K E\left(K_{s}, M\right)$ & encrypt $M$ using $K_{s}$ & $S K D\left(K_{s}, C\right)$ & decrypt $C$ using $K_{s}$ \\
\hline$S I G N(A, M)$ & sign of $M$ by $A$ & $S I G V(A, M)$ & verify $M$ signed by $A$ \\
\hline \multicolumn{4}{|c}{ TABLE II } \\
SYSTEM FUNCTIONS.
\end{tabular}

Some cryptographic functions run much slower than others. As some smart grid operations are time sensitive, it is very crucial to identify efficient cryptographic functions appropriately to protect the communication. To further understand the computational time of the cryptographic functions on computationally constrained devices, we measure the time needed to execute some representative cryptographic functions on Raspberry $\mathrm{Pi}$. Raspberry $\mathrm{Pi}$ is a tiny computer with a size similar to a credit card. The CPU is $700 \mathrm{MHz}$ and the memory available is $512 \mathrm{MB}$. Due to space limitation, we only present some of the results. More details can be found in [45].

Table III presents the time needed to create an RSA (the Rivest-Shamir-Adleman cryptosystem) signature and verify an RSA signature using different key sizes. The time spent on encrypting a message using public key is similar to the time

\begin{tabular}{l||l|l|l||l|l|l}
\multicolumn{1}{c||}{ RSA } & \multicolumn{3}{c||}{1024 bits } & \multicolumn{3}{c}{3072 bits } \\
\hline $\begin{array}{l}\text { Message } \\
\text { Size (bits) }\end{array}$ & $\begin{array}{l}\text { Sign. } \\
(\mathrm{ms})\end{array}$ & $\begin{array}{l}\text { Ver. } \\
(\mathrm{ms})\end{array}$ & $\begin{array}{l}\text { sign/ver } \\
\text { ratio }\end{array}$ & $\begin{array}{l}\text { Sign. } \\
(\mathrm{ms})\end{array}$ & $\begin{array}{l}\text { Ver. } \\
(\mathrm{ms})\end{array}$ & $\begin{array}{l}\text { sign/ver } \\
\text { ratio }\end{array}$ \\
\hline \hline 128 & 64.01 & 3.91 & 15.12 & 1048.37 & 11.49 & 91.23 \\
\hline 256 & 64.97 & 4.01 & 16.19 & 1033.46 & 11.65 & 88.68 \\
\hline 512 & 64.27 & 4.00 & 16.08 & 1047.96 & 11.69 & 89.67 \\
\hline \multicolumn{6}{c}{ TABLE III } \\
\hline \multicolumn{1}{c}{ RSA COMPUTATIONAL TIME. }
\end{tabular}

needed in verifying a signature. The time needed on decrypting a message using private key is similar to the time needed on signature creation. It can be observed that the time needed does not grow with message size but with key size. Column ratio in the table gives the time ratio of $\frac{\text { signature computation }}{\text { signature verification }}$. The time spent on a private key operation (signing a message) is much longer than that on a public key operation (verifying a signature). An efficient protocol should not require MDs to sign a lot of messages, especially when a long RSA key is used.

We also measured the time needed to generate different Diffie-Hellman keys with different key sizes [45]. A DH shared key generation is more expensive than an RSA signature verification. It implies that it may not be appropriate to re-generate DH shared key for each data collection instance. By adopting different cryptographic functions and techniques carefully based on their security features and computational complexities, our protocol facilitates efficient and secure data collection.

\section{Protocol Overview}

To detect replay attacks, we adopt a similar way as the widely used Kerberos protocol (RFC4120) that uses timestamps. The parties who talk directly should first synchronize their clocks. When a timestamp is included in a message, the receiver should check whether the carried timestamp is within a certain amount of difference from its local clock. The default threshold in Kerberos is 300 seconds. In our protocol, the threshold would depend on the expected delay in transmitting the message and granularity of clock synchronization.

Because encrypting data using public key cryptography is very expensive, before any data collection, we should first develop shared keys among PO, DCs, and MDs for data protection. To ensure data reported by a certain MD can be decrypted by the PO only, we need to establish a key that is known by PO and that MD. We call a key that is known by exactly two parties a pairwise shared key. PO and each DC should also develop a pairwise shared key to protect their conversations. The same applies to DC with each MD it will talk to. Apart from pairwise keys, to facilitate a certain command or instruction to be delivered to a group of MDs in a secure and efficient manner, we also develop a set of group keys that each group key is shared between the PO, a DC, and the MDs that connect to that DC.

The PO initiates the Shared Key Generation Process to establish the necessary pairwise shared keys and group keys. We adopt the Diffie-Hellman key exchange mechanism to develop all pairwise shared keys. We authenticate the DH half 
keys using the long-term public keys to avoid the man-inthe-middle attack. Once the pairwise shared keys and group keys are established, they will be used for data collection and command delivery.

As DH operations are expensive, we should not re-generate the DH shared keys for every data collection. However, it may not be very secure if we use the same shared keys to encrypt data collected at different times. To strike a balance of computational complexity and security, the data encryption key for each data collection instance depends on both the DH shared key and the timestamp. As the timestamp changes for every data collection instance, the data encryption key will be changed even though we do not re-generate the $\mathrm{DH}$ shared key. In the following, we will first describe the Shared Key Generation process in Section IV. The detailed message exchanges of the four operations mentioned in Section III-A will be provided in Section V.

\section{Shared Key Generation}

Let the set of MDs be $\mathbb{M D}$ and the set of DCs be $\mathbb{D C}$. Before the PO initiates the process, $\mathrm{PO}$ has to assign a set of MDs for DC to connect to. We let $M D L I S T_{i} \subseteq \mathbb{M D}$ be the set of

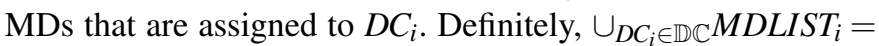
$\mathbb{M D}$. However, $M D L I S T_{i} \cap M D L I S T_{j}$, where $i \neq j$, may not necessarily be $\emptyset$. It is possible that PO would like multiple DCs to collect data from the same MD to enhance reliability. In fact, different assignments between MDs and DCs would differ in data security, cost, and data collection time. In Section VI, we will formulate the assignment problem to minimize the data collection time.

In the rest of this paper, for the ease of discussion, we use shared key to refer to pairwise shared key. We further denote $K_{B}^{A}$ as the shared key between $A$ and $B$. We refer to the set $\left\{P O, D C_{i}\right\} \cup M D L I S T_{i}$ as group $G_{i}$, and the group key of $G_{i}$ is $G K_{i}$. We use $M 1|| M 2$ to represent concatenating messages $M 1$ and $M 2$. The definitions of the functions used can be found in Table II.

Figure 2 presents a summary of the initial shared key generation process. When the procedure starts, the only keys an MD or a DC knows are its own public/private keys and the public key of the PO. After the procedure, $M D_{j}$ should have established $K_{M D_{j}}^{P O}, K_{M D_{j}}^{D C_{i}}$, and $G K_{i}$ if $M D_{j} \in M D L I S T_{i}$. Through the procedure, $D C_{i}$ knows $G K_{i}, K_{D C_{i}}^{P O}$ and $K_{M D_{j}}^{D C_{i}}$ for all $M D_{j} \in M D L I S T_{i}$. The detailed procedure is as follows:

1) $\mathrm{PO}$ starts the key generation process. It first generates a DH secret $a$ to talk to the DCs. PO also captures the current timestamp $T 1$ and sends the following message to $D C_{i} . T 1$ should be kept until the whole key generation process is done.

$$
P O \rightarrow D C_{i}: P K E\left(D C_{i}^{+}, g^{a} \| T 1\right), \operatorname{SIGN}\left(P O, g^{a} \| T 1\right)
$$

$g^{a}$ is encrypted and so an eavesdropper cannot read $g^{a}$. Because PO signs $g^{a} \| T 1$ and $T 1$ is a timestamp, an attacker cannot change $T 1$ or $g^{a}$ easily without being detected. Suppose an attacker also knows $D C_{i}^{+}$, although he can create $\operatorname{PKE}\left(D C_{i}^{+}, g^{a^{\prime}} \| T 1^{\prime}\right)$ using his own $g^{a^{\prime}}$ and $T 1^{\prime}$, he cannot forge the signature $\operatorname{SIGN}\left(P O, g^{a}|| T 1\right)$. To further enhance

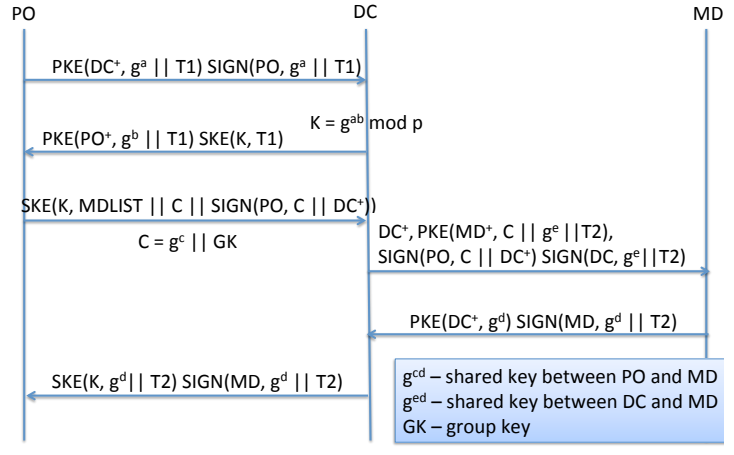

Fig. 2. Initial Shared Key Generation.

security, PO can use different $a$ 's for different DCs, but it has to generate different signatures for different $a$ 's and remember which is used for which DC.

2) When $D C_{i}$ receives the message, it uses $D C_{i}^{-}$to retrieve $g^{a}$ and $T 1$. It checks whether the received signature $\operatorname{SIGN}\left(P O, g^{a} \| T 1\right)$ is correct. If so, $D C_{i}$ checks whether $T 1$ is within an acceptable range. If so, it generates its DH secret $b$ and computes $K$ as $g^{a b}$ mod $p . K$ is then the shared key between $P O$ and $D C_{i}\left(K_{D C_{i}}^{P O}\right)$. It encrypts its publich DH key $\left(g^{b}\right)$ using PO's public key and send it to PO. It also proves it knows $K$ by providing $S K E(K, T 1)$.

$$
D C_{i} \rightarrow P O: P K E\left(P O^{+}, g^{b} \| T 1\right), \operatorname{SKE}(K, T 1)
$$

The timestamp $T 1$ is used to detect replay attack as mentioned in Section III-D. When a message is accepted, $D C_{i}$ should record $T 1$. When another message arrives that carries a time stamp $T, T$ is accepted only when it is not exactly the same as $T 1$ and the time difference between $T$ and it local clock is acceptable. Note that an attacker, who does not know $D C_{i}^{-}$, cannot retrieve $g^{a}$ and $T 1$ from $P K E\left(D C_{i}^{+}, g^{a} \| T 1\right)$. Therefore, when PO receives a correct reply, he knows that it was $D C_{i}$ who sent him the message.

3) When PO receives the message, it can retrieve $g^{b}$ using $P O^{-}$to compute $K$. It then uses $K$ to decrypt $\operatorname{SKE}(K, T 1)$ and retrieves $T 1$. If this is the same as the one he sent earlier, PO can confirm that it was $D C_{i}$ who sent the message. It then sends $D C_{i}$ the list of MDs, together with the MDs' public keys, that it assigns $D C_{i}$ to talk to. It also creates $C$ for $D C_{i}$ to talk to the MDs in the list. $C$ contains $g^{c}$, which is used for establishing shared keys between PO and MDs, and $G K_{i}$, which is the group key of $G_{i}$. The public keys of the MDs should also be sent (We assume they are included in MDLIST in Figure 2).

$P O \rightarrow D C_{i}: S K E\left(K, M D L I S T_{i}|| C|| S I G N\left(P O, C \| D C_{i}^{+}\right)\right)$where $C=g^{c} \| G K_{i}$

It is worth noting that $\mathrm{PO}$ also sends $\operatorname{SIGN}\left(P O, C \| D C_{i}^{+}\right)$and further encrypts it using $K$. This allows $D C_{i}$ to detect whether the message has been tampered. As $C$ is encrypted using $K$, it should be safe from eavesdroppers. $G K_{i}$, which should be known to PO, $D C_{i}$, and those MD in MDLIST $T_{i}$, is protected then.

4) When $D C_{i}$ receives the message, it first uses $K$ to retrieve $C$ and $\operatorname{SIGN}\left(P O, C|| D C_{i}^{+}\right)$. It verifies whether the signature is correct. If so, $D C_{i}$ can then generate its DH half key, $g^{e_{i}}$ 
for establishing shared keys with the MDs. $D C_{i}$ also captures the current timestamp $T 2$, which must be larger than $T 1$, and sends the information to $M D_{j}$ in $M D L I S T_{i}$ using the public keys provided. $D C_{i}$ also needs to send its public key. To allow $M D_{j}$ to detect whether the message has been tampered, two signatures, $\operatorname{SIGN}\left(P O, C \| D C^{+}\right)$and $\operatorname{SIGN}\left(D C_{i}, g^{e^{i}} \| T 2\right)$, are sent as well.

$$
\begin{gathered}
D C_{i} \rightarrow M D_{j}: D C_{i}^{+}, \operatorname{PKE}\left(M D_{j}^{+}, C\left\|g^{e_{i}}\right\| T 2\right), \\
\operatorname{SIGN}\left(P O, C \| D C^{+}\right), \operatorname{SIGN}\left(D C_{i}, g^{e_{i}} \| T 2\right)
\end{gathered}
$$

As $D C_{i}^{+}$is sent in plaintext and $C\left\|g^{e_{i}}\right\| T 2$ is encrypted using the public key of $M D_{j}$, it is possible for an attacker to create its own $D C_{i}^{+}, P K E\left(M D_{j}^{+}, C\left\|g^{e_{i}}\right\| T 2\right)$ and $\operatorname{SIGN}\left(D C_{i}, g^{e_{i}} \| T 2\right)$. That is, let the attacker be $A K$. He can send $A K^{+}, P K E\left(M D_{j}^{+}, C^{\prime}\left\|g_{a k}^{e_{i}}\right\| T 2^{\prime}\right)$ and $\operatorname{SIGN}\left(A K, g_{a k}^{e_{i}} \| T 2^{\prime}\right)$ to $M D_{j}$, trying to pretend to be $D C_{i}$. However, he cannot forge PO to create $\operatorname{SIGN}\left(P O, C^{\prime} \| A K^{+}\right)$to cheat $M D_{j}$. This message is thus safe from message tampering and an attacker cannot impersonate $D C_{i}$.

5) Upon receiving the message, $M D_{j}$ first decrypts $\operatorname{PKE}\left(M D_{j}^{+}, C\left\|g^{e_{i}}\right\| T 2\right)$ using his private key to retrieve $C\left\|g^{e_{i}}\right\| T 2$. It then verifies the two signatures to ensure the message has not been tampered. It should also check whether $T 2$ is acceptable in a similar way that $D C_{i}$ verifies $T 1$ to detect replay attacks. If the message passes the tests, $M D_{j}$ creates a DH secret key $d$ to establish the shared key between itself and PO $\left(K_{M D_{j}}^{P O}\right)$, which is $g^{c d}$, and the shared key with $D C_{i}\left(K_{M D_{j}}^{D C_{i}}\right)$, which is $g^{e_{i} d}$. It then encrypts $g^{d}$ using the public key of $D C_{i}$ so that $g^{d}$ is safe from eavesdroppers. It also signs $g^{d}$ and $T 2$ to defend against impersonation and replay attacks.

$$
M D_{j} \rightarrow D C_{i}: \operatorname{PKE}\left(D C_{i}^{+}, g^{d}\right), \operatorname{SIGN}\left(M D_{j}, g^{d} \| T 2\right)
$$

If an attacker eavesdropped an earlier communication, it cannot simply replay the message from the previous session because $T 2$ carried in the new message should be different. By signing $g^{d}$, we can defend against attackers who want to impersonate $M D_{j}$ in replying to $D C_{i}$.

6) When $D C_{i}$ receives the message, it decrypts $P K E\left(D C_{i}^{+}, g^{d}\right)$ using its private key and retrieves $g^{d}$. It can then verify the signature to detect whether the message has been tampered. If not, it sends $g^{d}$ to PO by encrypting it using $K$. It also sends the signature by $M D_{j}$ it received to PO.

$$
D C_{i} \rightarrow P O: \operatorname{SKE}\left(K, g^{d} \| T 2\right), \operatorname{SIGN}\left(M D_{j}, g^{d} \| T 2\right)
$$

As only $D C_{i}$ and PO know $K$, only PO can read $g^{d}$ in $\operatorname{SKE}\left(K, g^{d} \| T 2\right)$. By checking whether $T 2$ is later than $T 1$ kept in memory, PO can detect whether it is a replay. The signature of $M D_{j}$ on $g^{d}$ and $T 2$ authenticates that it was $M D_{j}$ who created $g^{d}$.

7) If $g^{d} \| T 2$ encrypted using $K$ and signed by $M D_{j}$ are the same, PO can assume the message has not been tampered. PO can then compute $K_{M D_{j}}^{P O}$ to be $g^{c d}$. Note that as $D C_{i}$ can only read $g^{c}$ and $g^{d}$ but neither $c$ nor $d$, it cannot compute $g^{c d} \cdot g^{c d}$ is thus a key shared by $P O$ and $M D_{j}$ only.

We now analyze the memory needed for each entity to keep the shared keys. The PO needs to keep a shared key for each
DC, a shared key for each MD, and a group key for each group. The total number of keys is $2 \mathrm{x}|\mathbb{D C}|+|\mathbb{M D}| . D C_{i}$ has to keep $K_{D C_{i}}^{P O}$, a shared key with each MD belongs to its group, and a group key. The total is $2+\left|M D L I S T_{i}\right|$. For $M D_{j}$, for each group $G_{i}$ it belongs to, it has to keep a shared key with $D C_{i}$ and the group key $G K_{i}$. It is worth noting that $M D_{j}$ can establish different shared keys with PO through different DCs. If PO provides different $g^{c}$ 's for different DCs, the shared keys developed via different DCs must be different. Even when PO provides the same $g^{c}$ through different DCs, $M D_{j}$ can also establish different shared keys by replying different $g^{d}$ 's for different DCs. Therefore, $M D_{j}$ has to keep at most $3 x$ number of groups it belongs to keys in total. PO decides how many groups an MD is associated with and can thus establish keys according to the memory available in different MDs.

\section{Data Collection AND Command Delivery}

\section{A. PO initiates Data Collection of a Group of or All MDs}

It is a regular data collection initiated by the PO. We want the data collection to be secure, scalable, and efficient. To ensure data confidentiality and integrity, data reported by $M D_{j}$ is encrypted using $K_{M D_{j}}^{P O}$, a key shared by the PO and $M D_{j}$ only. Our data collection protocol is scalable because a single DC would collect data from multiple MDs. PO no longer needs to establish a single session to each MD. To acheive efficiency, we do not require computationally-constrained MDs to perform a lot of expensive operations. We also reduce the number of messages exchanged. To further reduce the time of data collection, we study how to assign DCs to collect data from the MDs in Section VI. In the following, we first present the data collection procedure in a step by step manner. Fig. 3 shows the whole process. In the figure, $K 1, K 2$, and $K 3$ are $K_{D C_{i}}^{P O}, K_{M D_{j}}^{P O}$, and $K_{M D_{j}}^{D C_{i}}$, respectively.

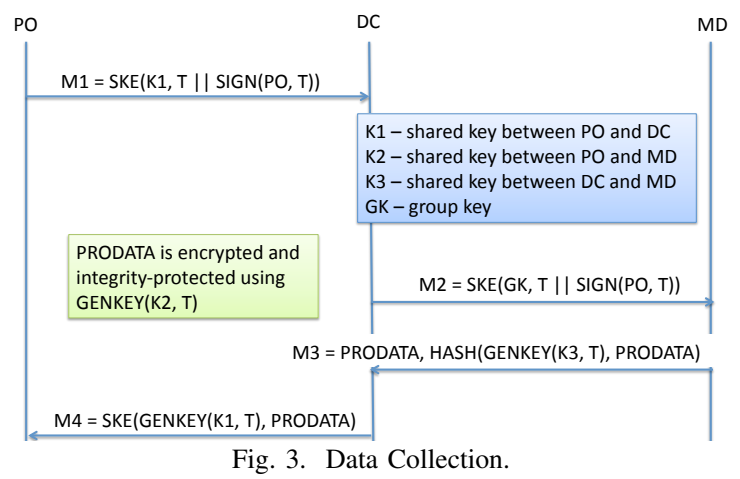

1) PO first identifies all the DCs to talk to according to a certain optimization criterion. It captures the current timestamp $T$, signs it, encrypts $T$ and the signature using $K_{D C_{i}}^{P O}$, and sends the encrypted message to $D C_{i}$. Note that it is possible that PO does not want to collect data from some MDs in MDLIST $T_{i}$ If so, PO should also include the list of intended MDs. We omit that in our protocol to simplify the discussion.

$$
P O \rightarrow D C_{i}: \operatorname{SKE}\left(K_{D C_{i}}^{P O}, T \| \operatorname{SIGN}(P O, T)\right)
$$

2) Upon receiving the message, $D C_{i}$ can retrieve $T \| \operatorname{SIGN}(P O, T))$ using the shared key. It first verifies 
the signature to ensure the message has not been tampered. To detect whether the message is a replayed one, it checks whether $T$ is acceptable. It then encrypts $T \| \operatorname{SIGN}(P O, T))$ using the group key $G K_{i}$ and sends the message to $M D_{j} \in M D L I S T_{i}$ (or only the MDs PO wants to collect data from).

$$
D C_{i} \rightarrow M D_{j}: \operatorname{SKE}\left(G K_{i}, T|| \operatorname{SIGN}(P O, T)\right)
$$

By encrypting the message using the group key, $D C_{i}$ only needs to create a single message for all MDs in its group. However, the group key cannot authenticate it was PO who requested the data collection because it is a key shared by many entities. We thus need to include a signature of PO to facilitate authentication. This message should work fine if $D C_{i}$ has to collect data from every MD in MDLIST $T_{i}$. However, when some MDs are not supposed to report data, those are not reporting can also read $T$ in the message. As $T$ is only a timestamp and is not a secret, knowing $T$ would not allow MDs to launch any attack. However, if this is a serious concern, $D C_{i}$ can send $\operatorname{SKE}\left(K_{M D_{j}}^{D C_{i}}, T \| S I G N(P O, T)\right)$ to the involved MDs instead. The disadvantage of this approach is $D C_{i}$ needs to create a different message for different MD and possibly incurs more delay in the data collection process. As an attacker does not know $G K_{i}$ and cannot forge $\operatorname{SIGN}(P O, T)$, the message is safe from impersonation.

3) When $M D_{j}$ receives the message, it retrieves the content using $G K_{i}$. It first verifies the signature and whether $T$ is within an acceptable range. If so, $M D_{j}$ generates keys for protecting the data and allows $D C_{i}$ to perform integrity check. Let the Message Key (MK) be $M K=\operatorname{GENKEY}\left(K_{M D_{j}}^{P O}, T\right)$. An encryption key and an integrity key developed based on $M K$ are used to protect the data. The protected data is denoted as PRODATA. As $M K$ depends on $T$, different $M K$ 's will be used for different data collection instances even $K_{M D_{j}}^{P O}$ is not changed. $M D_{j}$ also generates $D K=\operatorname{GENKEY}\left(K_{M D_{j}}^{D C_{j}}, T\right)$ to protect from message tampering. The hash of PRODATA using $D K$ is computed and sent to $D C_{i}$.

$$
M D_{j} \rightarrow D_{i} \text { : PRODATA, HASH (DK, PRODATA) }
$$

Note that as PRODATA is encrypted using a derivative of $K_{M D_{j}}^{P O}, D C_{i}$ cannot decrypt and read it. PRODATA is thus secure against honest-but-curious DCs. The hash of PRODATA, on the other hand, is computed using a derivative of $K_{M D_{j}}^{D C_{i}}$. $D C_{i}$ can thus check whether an attacker has tampered the message before relaying the data back to the PO. As an attacker does not know $K_{M D_{j}}^{D C_{i}}$, he cannot impersonate $M D_{j}$ to send $D C_{i}$ the data.

4) When the encrypted data arrives, $D C_{i}$ verifies the hash to ensure PRODATA was generated by $M D_{j}$ even it cannot decrypt PRODATA. It then forwards PRODATA to $P O$ by encrypting it $G E N K E Y\left(K_{D C_{i}}^{P O}, T\right)$. Alternatively, $D C_{i}$ can encrypt all the replies from $M D \mathrm{~s}$ in a single message. In this case, only a single symmetric key encryption is needed, but $P O$ may receive some data later.

$$
D C_{i} \rightarrow \text { PO: } \operatorname{SKE}\left(\operatorname{GENKEY}\left(K_{D C_{i}}^{P O}, T\right), \text { PRODATA }\right)
$$

5) Upon receiving the message, PO retrieves PRODATA by decrypting the message using $\operatorname{GENKEY}\left(K_{D C_{i}}^{P O}, T\right)$. It also de- velops $M K$ to extract the data from PRODATA. Because $K_{D C_{i}}^{P O}$ is a shared secret between $\mathrm{PO}$ and $D C_{i}$, an attacker cannot forge the message. If the message is tampered, say, a bit is flipped, PRODATA decrypted would be scrambled and would not pass the integrity check using $M K$. The data sent from $M D_{j}$ are thus remain confidential and secure.

It can be observed that each MD, each DC, and the PO need to perform one public key operation only no matter how many messages it has to handle. Besides, the signature verification that MDs and DCs have to perform is not very expensive when compared with signature creation. Our protocol is thus very light-weight and scalable.

\section{B. $P O$ requests data from $M D_{j}$}

We list the steps PO can take to request date from $M D_{j}$ :

1) $\mathrm{PO}$ first identifies a certain $D C_{i}$ such that $M D_{j} \in G_{i} . T$ is the timestamp. Apart from signing the timestamp, $P O$ also encrypts the timestamp using $K_{M D_{j}}^{P O}$.

$$
P O \rightarrow D C_{i}: \operatorname{SKE}\left(K_{D C_{i}}^{P O}, T\|\operatorname{SIGN}(P O, T)\| \operatorname{SKE}\left(K_{M D_{j}}^{P O}, T\right)\right)
$$

2) $D C_{i}$ sends the information to $M D_{j}$ after verifying the signature on $T$.

$$
D C_{i} \rightarrow M D_{j}: \operatorname{SKE}\left(K_{M D_{j}}^{D C_{i}}, T \| \operatorname{SKE}\left(K_{M D_{j}}^{P O}, T\right)\right)
$$

Steps 3 - 5 are the same as in Section V-A.

Similar mechanism can be used for PO to issue an urgent command to $M D_{j} . M D_{j}$ should respond with an acknowledgement instead of PRODATA.

\section{C. $M D_{j}$ initiates an urgent data report}

The following are the steps by $M D_{j}$ to report unsolicited urgent data:

1) $M D_{j}$ first identifies a certain $D C_{i}$ to relay the message and records the current timestamp T. PRODATA and DK are generated as in Step 3 in Section V-A.

$$
\begin{gathered}
M D_{j} \rightarrow D C_{i}: \\
\left.\operatorname{SKE}\left(K_{M D_{j}}^{D C_{i}}, T \| \text { PRODATA } \| \text { HASH (DK, PRODATA }\right)\right)
\end{gathered}
$$

2) $D C_{i}$ verifies the hash and forwards PRODATA to $P O$.

$$
D C_{i} \rightarrow P O: S K E\left(K_{D C}^{P O}, T \| P R O D A T A\right)
$$

3) $P O$ can then extract $T$ using $K_{D C_{i}}^{P O}$ to develop the appropriate keys to decrypt PRODATA.

In reporting emergency information, latency and reliability are very important. In the protocol, $M D_{j}$ does not need to perform any expensive public key operation before sending the data report. The latency is thus very small. To enhance reliability, $M D_{j}$ can send the data to $P O$ via multiple $D C$ s. It has to compute $H A S H(D K, P R O D A T A)$ and encrypt T||PRODATA||HASH(DK, PRODATA) using different keys for different $D C$ s in Step 1. As both operations are not expensive, $M D_{j}$ can send out the reports promptly.

\section{PO issues an urgent command to a group of MDs}

When PO invoke a group of MDs, it employs the following:

1) Similar to requesting data, $P O$ should first identify the $D C$ s that cover all the MDs that it wants to send the urgent 
command to. Let the command be COMD. MDLIST $T_{i}$ contains the $M D$ s that $D C_{i}$ should talk to.

$$
\begin{gathered}
P O \rightarrow D C_{i}: \\
\operatorname{SKE}\left(K_{D C_{i}}^{P O}, \operatorname{SIGN}(P O, C O M D)\left\|M D L I S T_{i}\right\| C O M D\right)
\end{gathered}
$$

2) $D C_{i}$ sends to each $M D_{j}$ in $M D L I S T_{i}$ the urgent command.

$$
D C_{i} \rightarrow M D_{j}: \operatorname{SKE}\left(G K_{i}, \operatorname{SIGN}(P O, C O M D) \| C O M D\right)
$$

The signature of the command by the PO provides authentication check to all MDs and DCs. By using a group key in Step 2, we share the same issue as in Step 2 of Section V-A. The administrator can thus select the most appropriate way to strike a balance of security and efficiency.

\section{GROUPING OptIMIZATION}

\section{A. Deriving the Optimized Data Collection Time}

We now consider how to minimize the time to perform data collection from a group of MDs by selecting a single appropriate DC to collect data from each MD. To compute the total time needed for PO to collect the data, we first define some notations to represent the time needed to perform a single cryptographic operation defined in Table II. Theoretically speaking, the time needed for a cryptographic operation depends on the size of the message. As we only perform public key operations on small-sized messages, we ignore this factor and denote $T^{p}(O P, A)$ as the time needed for $A$ to execute public key cryptographic operation PKE, PKD, SIGN, and $S I G V$. For example, the time for PO to sign a message is $T^{p}(S I G N, P O)$. To capture the effect of message size on the computational time of symmetric key and hash operations, we denote the time needed as $T^{s}(O P, A, S I Z E)$. As symmetric key encryption and decryption take roughly the same time, we use $S K$ to represent both $S K E$ and $S K D$. We also use HASH to denote both hash computation and verification. To simplify our discussion, we assume the size of $T \| \operatorname{SIGN}(P O, T)$ in Section $\mathrm{V}-\mathrm{A}$ as 1 unit. That is, the time needed for $D C_{i}$ to develop message $\operatorname{SKE}\left(G K_{i}, T \| \operatorname{SIGN}(P O, T)\right)$ is $T^{s}\left(S K, D C_{i}, 1\right)$. The one-way network delay between $A$ and $B$ is $T^{n}(A, B)$. We also let $x_{i j}=1$ if $M D_{j}$ belongs to $G_{i}$.

To simplify our discussion, we use $M 1, M 2, M 3$, and $M 4$ to represent the four messages exchanged between PO, DCs, and MDs as shown in Fig. 3. We only consider the situation where a $D C$ reports all data collected in a single message to PO. To illustrate the process of time analysis, we present Fig. 4 to explain the different time components in the whole data collection process. In the picture, we assume there are only two MDs.

We first develop the time needed for $D C_{i}$, after having prepared $M 2$, to send message $M 2=\operatorname{SKE}\left(G K_{i}, T \| \operatorname{SIGN}(P O, T)\right)$ to $M D_{j}$ and verify the hash of $M D_{j}$ 's reply, which is denoted as $T_{i j} . T_{i j}$ is the sum of the following components:

1) round-trip network delay between $D C_{i}$ and $M D_{j}$ : $2 T^{n}\left(D C_{i}, M D_{j}\right)$

2) time needed for $M D_{j}$ to generate reply $M 3$ (Step 3): $T^{s}\left(S K, M D_{j}, 1\right)+T^{p}\left(S I G V, M D_{j}\right)+T^{s}\left(S K, M D_{j}\right.$, size $)+$ $2 T^{s}\left(H A S H, M D_{j}\right.$, size $)$ where size is the size of the data in terms of number of units.

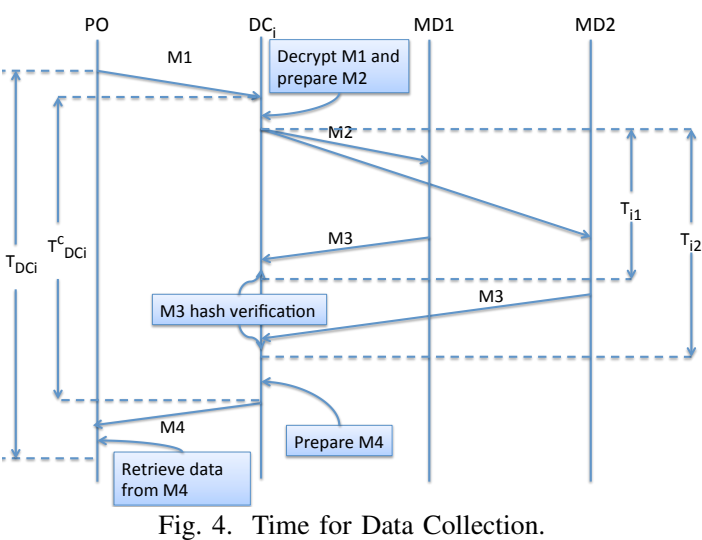

3) time needed for $D C_{i}$ to verify the hash: $T^{s}\left(H A S H, D C_{i}\right.$, size $)$

Before $D C_{i}$ can send message $M 2=$ $\operatorname{SKE}\left(G K_{i}, T \| \operatorname{SIGN}(P O, T)\right)$ to $M D_{j}, D C_{i}$ needs to decrypt $M 1$ and prepare $M 2$. As described in Step 2 in Section V-A, $D C_{i}$ has to spend $2 T^{s}\left(S K, D C_{i}, 1\right)+T^{p}\left(S I G V, D C_{i}\right)$ time to prepare $M 2$. We now study the time needed for $D C_{i}$ to prepare the reply (M4) to PO after verifying the hashes of the replies from all MDs. Let $N_{i}$ be $\sum_{j} x_{i j}$. That is, $N_{i}$ is the number of MDs in $G_{i}$. The total amount of data received by $D C_{i}$ is $N_{i} \times$ size. The time to prepare $M 4$ is $T^{s}\left(S K, D C_{i}, N_{i} \times\right.$ size $)$. Therefore, the total time needed for $D C_{i}$ from the moment it receives $M 1$ from $P O$ to the moment it sends out $M 4$ to the $\mathrm{PO}$ is:

$$
\begin{aligned}
T_{D C_{i}}^{c}= & 2 T^{s}\left(S K, D C_{i}, 1\right)+T^{p}\left(S I G V, D C_{i}\right)+\max _{j}\left\{x_{i j} T_{i j}\right\} \\
& +T^{s}\left(S K, D C_{i}, N_{i} \times \text { size }\right)
\end{aligned}
$$

We now study the time from the moment that PO sends out $M 1$ until the moment that PO successfully decrypts and verifies the data carried in $M 4$ sent by $D C_{i}$. We denote this time as $T_{D C_{i}}$. To retrieve the raw data from $M 4=$ $\operatorname{SKE}\left(\operatorname{GENKEY}\left(K_{D C_{i}}^{P O}, T\right)\right.$, PRODATA $)$, PO first needs to decrypt $M 4$ using $G E N K E Y\left(K_{D C_{i}}^{P O}, T\right)$. It then needs to decrypt and verify the hash carried in PRODATA. Therefore, $T_{D C_{i}}$ is

$$
\begin{aligned}
T_{D C_{i}}= & 2 T^{n}\left(P O, D C_{i}\right)+T_{D C_{i}}^{c}+ \\
& 2 T^{s}\left(S K, P O, N_{i} \times \text { size }\right)+T^{s}\left(H A S H, P O, N_{i} \times \text { size }\right) \\
= & f(i)+\max _{j}\left\{x_{i j} T_{i j}\right\}
\end{aligned}
$$

where

$$
\begin{aligned}
f(i)= & 2 T^{s}\left(S K, D C_{i}, 1\right)+T^{p}\left(S I G V, D C_{i}\right)+2 T^{n}\left(P O, D C_{i}\right) \\
& +2 T^{s}\left(S K, P O, N_{i} \times \text { size }\right)+T^{s}\left(H A S H, P O, N_{i} \times \text { size }\right)
\end{aligned}
$$

\section{B. Problem Formulation}

When $P O$ wants to collect all data as soon as possible, we should assign each $M D$ to an appropriate $D C$ such that the maximum $T_{D C_{i}}$ over all $i \in \mathbb{D}$ is minimized. Such an objective leads to what is known in the literature as a minimax problem. From Equation 1, we can simplify the terms into two major categories for the minimax optimization: One is the maximizing component $\left(\max _{j}\left\{x_{i j} T_{i j}\right\}\right)$ and the other is the summative part $(f(i))$. The former consists mostly of the network delay whose maximum value will determine 
the total completion time for data collection by a DC. The latter includes the processing time, including the cryptographic computation, whose total time will be a summation operation. In what follows, we will ignore the maximization components, as it is rather trivial to address alone, and concentrate on the summative part. Under a realistic data collection scenario, summative component will likely be the dominant term to determine the overall performance.

When the summative part is considered, the problem looks very similar to the makespan minimization problem from the scheduling theory [46], [47]. Scheduling theory considers problems where a set of jobs (tasks) are to be assigned to a set of machines or processors to satisfy an objective. One machine can only work on one job at a time. The well-established 3-field classification introduced in [48] uses $\alpha|\beta| \gamma$ notation, where job, machine, and scheduling characteristics are denoted by $\alpha, \beta$, and $\gamma$, respectively. The summative part of our objective function is denoted by $Q \| C_{\max }$, where arbitrary number of machines operating at different speeds must be used to complete a given set of tasks with the minimum makespan objective. This problem setting is also known in the literature as uniform parallel machines [49]. In our problem, machines are DCs, and tasks are MDs whose data need to be collected.

The Integer Linear Programming (ILP) formulation for our summative part may be formulated as follows:

$$
\begin{aligned}
\min \max & \sum_{j} x_{i j} t_{i j} \\
\text { s.t. } & \sum_{j} x_{i j}=1, \quad \forall i \in \mathbb{D} \\
& x_{i j} \in\{0,1\} \quad \forall i \in \mathbb{D}, \forall j \in \mathbb{M}
\end{aligned}
$$

where $x_{i j}$ represents whether DC $i$ is assigned to collect data from MD $j$, and $t_{i j}$ is the amount of the summative part of the total data collection time of MD $j$ 's data by DC $i$.

When we let $C_{\max }$ represent the maximum data collection time, the above formulation can be rewritten in a standard form as follows:

$$
\begin{aligned}
\min & C_{\max } \\
\text { s.t. } & \sum_{j} x_{i j}=1, \quad \forall i \in \mathbb{D} \\
& \sum_{j} x_{i j} t_{i j} \leq C_{\max }, \quad \forall i \in \mathbb{D} \\
& x_{i j} \in\{0,1\} \quad \forall i \in \mathbb{D}, \forall j \in \mathbb{M}
\end{aligned}
$$

The above problem can be shown to be strongly NPHard [50], [51] by a reduction from a 3-Partition problem [52]. Also note that this problem is a kind of the dual of the bin packing problem [50], [53].

As solving the ILP of minimum makespan is NP-hard by reduction from a 3-Partition problem, and thus making it unlikely that a polynomial algorithm exists, we develop a greedy heuristic, Least Loaded DC First $\left(\mathrm{L}^{3} \mathrm{~F}\right)$, to solve the problem. We find the largest time for data collection for any $(D C, M D)$ pair, say $\delta, \mu$. We assign MD $\mu$ to a $D C$ that will complete in the least time. Next, we pick the next largest time

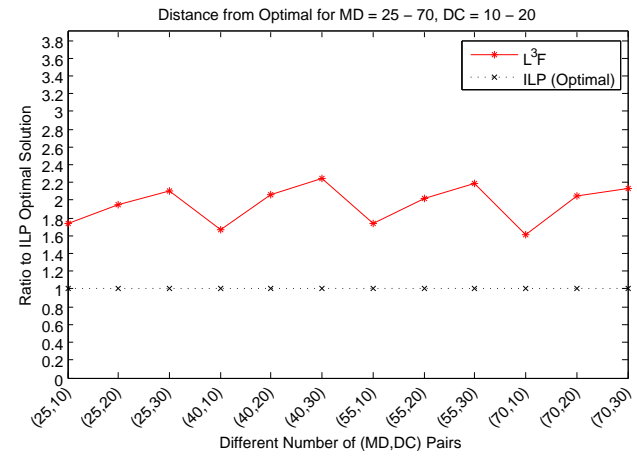

Fig. 5. Ratio of total data collection time for $\mathrm{L}^{3} \mathrm{~F}$ to Optimal ILP.

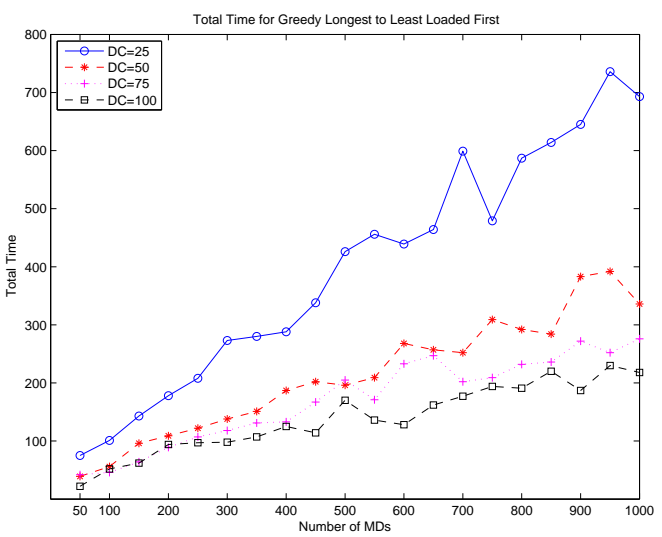

Fig. 6. Performance of $\mathrm{L}^{3} \mathrm{~F}$ in terms of total data collection time over changing the number of MDs with 4 different number of DCs.

and assign it to the least loaded DC for the corresponding MD. We iterate until we deplete unassigned MDs. It is obvious that the complexity of the algorithm is $O(d)$. Due to the page limitation, we omit the details of the full algorithm and refer the reader to [45].

\section{ViI. Performance Evaluation}

We have used CPLEX to solve the ILP formulations and implemented our approaches in $\mathrm{C}++$. Since the problem is NP-Hard, the ILP formulation that can be solved by CPLEX hits a wall rather quickly: After about $70 \mathrm{MDs}$ and $35 \mathrm{DCs}$, CPLEX started taking very long to yield any results. Thus, we have run some simulations up to 70 MDs and 35 DCs each with 30 runs to get an idea of the comparative performance results. The time for collecting data from MDs by DCs are randomly generated from a uniform probability distribution in the range of 10 to 100 . The number of DCs took the values of 10,20, and 30 while the number of MDs were assigned $25,40,55$, and 70. All possible combinations were run for 30 times for statistical significance.

Figure 5 shows the performance of ILP and $\mathrm{L}^{3} \mathrm{~F}$ for all 12 combinations of the number of MDs and DCs. It plots the total time values returned by the ILP from CPLEX as the reference point and hence shows it as a straight line on bottom. $\mathrm{L}^{3} \mathrm{~F}$, 
being a greedy algorithm, performed worse with an average distance ratio to the optimum of approximately 1.96 .

For more MDs and DCs, ILP cannot yield results. Thus, we only report $\mathrm{L}^{3} \mathrm{~F}$ in extensive simulations with the number of MDs going up to 1000 in increments of 50 starting from 50 and number of DCs at 25, 50, 75, 100. We had a total of 80 unique $(M D, D C)$ pairs. Again, in order to attain statistical significance, each combination pair was run 30 times. The time values for the data collection from MDs by DCs were generated using a uniform density function in the range of 10 to 100 . Figure 6 displays the total time of data collection for $\mathrm{L}^{3} \mathrm{~F}$ over the number of MDs from 50 to 1000 for 25, 50, 75, and 100 DCs as separate lines. Except for when the number of DCs was equal to 25 , the total time increases with respect to larger number of MDs is with moderate slope. When DC is equal to 25 , the increase is rather steep but still linear. This behavior might indicate that when there is significant imbalance between the number of DCs and MDs the total time to collect data may adversely affected. This point of operating overload is hard to have a threshold value to associate with but nevertheless should be considered.

\section{CONCLUSION}

The bidirectional power and information flow of the Smart Grid vision has led to the proliferation of a variety of measurement devices. These devices generate unprecedented amounts of data. The existing, legacy protocols are not capable of addressing this new phenomenon. In order to address this challenge, we propose a comprehensive and secure communications protocol to enable a power operator to collect data from measurement devices in a practical, scalable, and efficient manner under a hierarchical data collection model. Intermediary nodes are assumed to follow the honest-butcurious model in relaying the data. Thus, our protocol paves the way for third party service provisioning, as envisioned by the NIST Smart Grid Framework. Examples of such services include outsourcing data collection by third party DCs, utilizing cloud computing services for data storage and processing, etc. We formulate an optimization problem for associating the intermediary relay nodes with measurement devices for data collection in order to minimize the total data collection time. The problem is intractable and thus we present a heuristic algorithm with good approximation and fast convergence.

\section{REFERENCES}

[1] N. Kayastha, D. Niyato, E. Hossain, and Z. Han, "Smart grid sensor data collection, communication, and networking: a tutorial," Wireless Communications and Mobile Computing, pp. n/a-n/a, 2012.

[2] National Institute of Standards and Technology. (2013, October) NIST Framework and Roadmap for Smart Grid Interoperability Standards, Release 3.0. Smart Grid Interoperability Panel (SGIP).

[3] X. Fang, S. Misra, G. Xue, and D. Yang, "Managing smart grid information in the cloud: opportunities, model, and applications," Network, IEEE, vol. 26, no. 4, pp. 32-38, July 2012.

[4] S. Bera, S. Misra, and J. Rodrigues, "Cloud computing applications for smart grid: A survey," IEEE Tran. on Par. and Dist. Sys., no. 99, 2014.

[5] R. Tabassum, K. Nahrstedt, E. Rogers, and K.-S. Lui, "SCAPACH: Scalable password-changing protocol for smart grid device authentication," in Proc. of Third International Workshop on Privacy, Security, and Trust in Mobile and Wireless Systems (MobiPST), 2013.
[6] RFC 5246, "The transport layer security (tls) protocol version 1.2," 2008

[7] Y.-J. Kim, V. Kolesnikov, and M. Thottan, "Resilient end-to-end message protection for large-scale cyber-physical system communications," in Smart Grid Communications (SmartGridComm), 2012 IEEE Third International Conference on, Nov 2012, pp. 193-198.

[8] IEEE 1815-2012, "Dnp3 secure authentication version 5," 2011.

[9] International Electrotechnical Commission's (IEC) Technical Committee 57 (TC57). (2003) IEC 61850, Power Utility Automation.

[10] W. Wang and Z. Lu, "Cyber security in the smart grid: Survey and challenges," Computer Networks, vol. 57, no. 5, pp. 1344 - 1371, 2013.

[11] Y.-J. Kim, V. Kolesnikov, H. Kim, and M. Thottan, "SSTP: a scalable and secure transport protocol for smart grid data collection," in Proc. of IEEE SmartGridComm, 2011.

[12] T. Khalifa, K. Naik, M. Alsabaan, A. Nayak, and N. Goel, "Transport protocol for smart grid infrastructure," in Proc. of IEEE International Conference on Ubiquitous and Future Networks, 2010.

[13] X. Long, D. Tipper, and Y. Qian, "An advanced key management scheme for secure smart grid communications," in Proc. of IEEE SmartGridComm, 2013.

[14] N. Liu, J. Chen, L. Zhu, J. Zhang, and Y. He, "A key management scheme for secure communications of advanced metering infrastructure in smart grid," IEEE Tran. on Ind. Elect., vol. 60, no. 10, 2013.

[15] D. Wu and C. Zhou, "Fault-tolerant and scalable key management for smart grid," IEEE Trans. on Smart Grid, vol. 2, no. 2, June 2011.

[16] M. M. Fouda, Z. M. Fadlullah, N. Kato, R. Lu, and X. Shen, "A lightweight message authentication scheme for smart grid communications," IEEE Tran. on Smart Grid, vol. 2, no. 4, Dec. 2011.

[17] C. Bekara, T. Luckenbach, and K. Bekara, "A privacy preserving and secure authentication protocol for the advanced metering infrastructure with non-repudiation service," in Proc. of ENERGY, 2012.

[18] Y. Law, G. Kounga, and A. Lo, "WAKE: Key management scheme for wide-area measurement systems in smart grid," IEEE Comm. Mag., Jan. 2013.

[19] N. Liu, J. Chen, L. Zhu, J. Zhang, and Y. He, "A key management scheme for secure communications of advanced metering infrastructure in smart grid," IEEE Tran. on Industrial Electronics, vol. 60, no. 10, pp. 4746-4756, Oct 2013.

[20] A. Seshadri, M. Luk, and A. Perrig, "Sake: Software attestation for key establishment in sensor networks," in Proc. of International Conference on Distributed Computing in Sensor Systems, 2008.

[21] H. Nicanfar and V. Leung, "Multilayer consensus ecc-based password authenticated key-exchange (mcepak) protocol for smart grid system," IEEE Tran. on Smart Grid, vol. 4, no. 1, 2013.

[22] A. Perrig, R. Szewczyk, J. D. Tygar, V. Wen, and D. E. Culler, "Spins: Security protocols for sensor networks," Wirel. Netw., vol. 8, no. 5, pp. 521-534, Sep. 2002.

[23] S. Uludag, S. Zeadally, and M. Badra, "Techniques, Taxonomy, and Challenges of Privacy Protection in Smart Grid," in Privacy in a Digital, Networked World. Springer London, Feb. 2015.

[24] W. He, X. Liu, H. Nguyen, K. Nahrstedt, and T. Abdelzaher, "Pda: Privacy-preserving data aggregation in wireless sensor networks," in IEEE INFOCOM 2007, May 2007, pp. 2045-2053.

[25] W. He, H. Nguyen, X. Liu, K. Nahrstedt, and T. Abdelzaher, "iPDA: An integrity-protecting private data aggregation scheme for wireless sensor networks," in IEEE MILCOM 2008, Nov 2008, pp. 1-7.

[26] T. Feng, C. Wang, W. Zhang, and L. Ruan, "Confidentiality protection for distributed sensor data aggregation," in IEEE INFOCOM, April 2008.

[27] M. Groat, W. He, and S. Forrest, "Kipda: k-indistinguishable privacypreserving data aggregation in wireless sensor networks," in IEEE INFOCOM, April 2011, pp. 2024-2032.

[28] Y. Yan, Y. Qian, and H. Sharif, "A secure and reliable in-network collaborative communication scheme for advanced metering infrastructure in smart grid," in IEEE WCNC, March 2011, pp. 909-914.

[29] K. Kursawe, G. Danezis, and M. Kohlweiss, "Privacy-friendly aggregation for the smart-grid," in Privacy Enhancing Technologies. Springer, 2011, vol. 6794, pp. 175-191.

[30] C. Rottondi, G. Verticale, and C. Krauss, "Distributed privacy-preserving aggregation of metering data in smart grids," IEEE JSAC, vol. 31, no. 7, pp. 1342-1354, July 2013.

[31] H. Nicanfar, A. Alasaad, P. Talebifard, and V. Leung, "Network coding based encryption system for advanced metering infrastructure," in IEEE ICCCN, July 2013, pp. 1-7. 
[32] D. Niyato and P. Wang, "Cooperative transmission for meter data collection in smart grid," Communications Magazine, IEEE, vol. 50, no. 4, pp. 90-97, April 2012.

[33] S. Shao, S. Guo, X. Qiu, L. Meng, Y. Jiao, and W. Wei, "Traffic scheduling for wireless meter data collection in smart grid communication network," in Computing, Communication and Networking Technologies (ICCCNT), 2014 International Conference on, July 2014, pp. 1-7.

[34] F. Li, B. Luo, and P. Liu, "Secure information aggregation for smart grids using homomorphic encryption," in Smart Grid Communications (SmartGridComm), 2010 First IEEE International Conference on, Oct 2010, pp. 327-332.

[35] N. Yukun, T. Xiaobin, C. Shi, W. Haifeng, Y. Kai, and B. Zhiyong, "A security privacy protection scheme for data collection of smart meters based on homomorphic encryption," in EUROCON, 2013 IEEE, July 2013, pp. 1401-1405

[36] J. Kamto, L. Qian, J. Fuller, J. Attia, and Y. Qian, "Key distribution and management for power aggregation and accountability in advance metering infrastructure," in Smart Grid Communications (SmartGridComm), 2012 IEEE Third International Conference on, Nov 2012, pp. 360-365.

[37] F. Li and B. Luo, "Preserving data integrity for smart grid data aggregation," in Smart Grid Communications (SmartGridComm), 2012 IEEE Third International Conference on, Nov 2012, pp. 366-371.

[38] T. Chim, S. Yiu, V. Li, C. Hui, and J. Zhong, "PRGA: Privacypreserving Recording amp; Gateway-assisted Authentication of Power Usage Information for Smart Grid," IEEE Tran. on Dependable and Secure Computing, vol. PP, p. 1, 2014.

[39] L. Chen, R. Lu, and Z. Cao, "PDAFT: A privacy-preserving data aggregation scheme with fault tolerance for smart grid communications," Peer-to-Peer Networking and Applications, pp. 1-11, 2014.

[40] J. Hur, "Attribute-based secure data sharing with hidden policies in smart grid," IEEE Tran. on Par. and Dist. Sys., vol. 24, no. 11, 2013.

[41] P. Li, S. Guo, and Z. Cheng, "Joint optimization of electricity and communication cost for meter data collection in smart grid," IEEE Tran. on Emerging Topics in Computing, vol. 1, no. 2, pp. 297-306, Dec 2013.

[42] P. Li and S. Guo, "Delay minimization for reliable data collection on overhead transmission lines in smart grid," in Computing, Communications and IT Applications Conference (ComComAp), 2013, April 2013, pp. 147-152.

[43] M. Qiu, H. Su, M. Chen, Z. Ming, and L. Yang, "Balance of security strength and energy for a pmu monitoring system in smart grid," Communications Magazine, IEEE, vol. 50, no. 5, pp. 142-149, May 2012.

[44] G. Dan, K.-S. Lui, R. Tabassum, Q. Zhu, and K. Nahrstedt, "SELINDA: A secure, scalable and light-weight data collection protocol for smart grids," in Proc. of IEEE SmartGridComm, 2013.

[45] S. Uludag, K.-S. Lui, W. Ren, and K. Nahrstedt, "Secure and Scalable Communications Protocol for Data Collection with Time Minimization in the Smart Grid," Department of Computer Science, University of Illinois at Urbana-Champaign, Tech. Rep. 2014-07-16, July 2014. [Online]. Available: http://hdl.handle.net/2142/49985

[46] J. Y.-T. Leung, Handbook of scheduling : algorithms, models, and performance analysis. Boca Raton, Fla.: Chapman \& Hall/CRC, 2004.

[47] M. Pinedo, Scheduling: Theory, Algorithms, and Systems. Springer, 2008.

[48] R. Graham, E. Lawler, J. Lenstra, and A. Kan, "Optimization and approximation in deterministic sequencing and scheduling: a survey," ser. Annals of Discrete Mathematics. Elsevier, 1979, vol. 5, no. 2, pp. 287 - 326. [Online]. Available: http://www.sciencedirect.com/science/article/pii/S016750600870356X

[49] P. Brucker, Scheduling Algorithms. Springer, 2007. [Online]. Available: http://books.google.com/books?id=FrUytMqlCv8C

[50] J. Y.-T. Leung, "Bin packing with restricted piece sizes," Information Processing Letters, vol. 31, no. 3, pp. $145-149,1989 . \quad$ [Online]. Available: http://www.sciencedirect.com/science/article/pii/0020019089902238

[51] D. S. Hochbaum and D. B. Shmoys, "Using dual approximation algorithms for scheduling problems theoretical and practical results," J. ACM, vol. 34, no. 1, pp. 144-162, Jan. 1987. [Online]. Available: http://doi.acm.org/10.1145/7531.7535

[52] M. R. Garey and D. S. Johnson, Computers and Intractability: A Guide to the Theory of NP-Completeness. New York, NY, USA: W. H. Freeman \& Co., 1979.

[53] E. Coffman, Jr., M. Garey, and D. Johnson, "An application of bin-packing to multiprocessor scheduling," SIAM Journal on
Computing, vol. 7, no. 1, pp. 1-17, 1978. [Online]. Available: http://epubs.siam.org/doi/abs/10.1137/0207001 